\title{
Korea Red Ginseng Alters Electroencephalogram Spectra of Sleep-Wake Stage in Rats
}

\author{
Yuan Ma*, Jae Soon Eun**, Jae-Hoon Cheong***, Dong-Kwon Rhee****, \\ Jin Tae Hong****** and Ki-Wan Oh******\# \\ *Research Institute of Veterinary Medicine, Chungbuk National University, Cheongju. 361-763, S. Korea \\ **College of Pharmacy, Woosuk University, Samrye. 565-701, S. Korea \\ ***College of Pharmacy, Sahmyook University, Seoul. 139-742, S. Korea \\ ****College of Pharmacy, Sungkyunkwan University, Su-Won. 440-746, S. Korea \\ *****College of Pharmacy, Chungbuk National University, Cheongju. 361-763, S. Korea
}

(Received July 30, Accepted August 28, 2008)

\begin{abstract}
The present investigation was performed to evaluate the homeostatic regulation of sleep architecture by the ethanol extract of Korea red ginseng (KRG), since the available data were often controversial. In addition, it was also interested in whether the sleep-wake stages were differently affected by low and high doses of KRG. Each adult Wistar male rat was implanted with a transmitter for recording EEG and activity via telemetry. After one week of surgery, polygraphic signs of undisturbed sleep-wake activities were recorded for $12 \mathrm{~h}$ (between 9:00 am and 9:00 pm) after KRG administration. KRG (10 and $100 \mathrm{mg} / \mathrm{kg}$ ) increased non-rapid eye movement (NREM) sleep as well as total sleep. The total percentages of wakefulness were decreased comparably. KRG $(10 \mathrm{mg} / \mathrm{kg})$ decreased the power density of the $\delta$-wave $(0.75$ $4.5 \mathrm{~Hz}$ ) and increased $\alpha$-wave $(8.0-13.0 \mathrm{~Hz})$ in the NREM and rapid eye movement (REM) sleep. KRG also decreased $\delta$-wave power density in wake time. However, KRG $(100 \mathrm{mg} / \mathrm{kg})$ increased $\delta$-wave and decreased $\theta$-wave $(5.0-9.0 \mathrm{~Hz})$ power density in wake time, while showed little effect on the power density in NREM and REM sleep. In conclusion, low and high doses of KRG increase spontaneous sleep and NREM sleep and differently regulate the EEG spectra in REM and NREM sleep.
\end{abstract}

Keywords : Korea red ginseng (KRG); sleep; electroencephalogram (EEG); non-rapid eye movement (NREM); rapid eye movement (REM); power density.

\section{INTRODUCTION}

A wide range of behavioral studies have provided considerable evidence that the regulation of the total amount of sleep is a homeostatic process ${ }^{1)}$. Homeostatic control mechanisms are activated to compensate for insufficient or excess sleep. In mammals, sleep consists of two major stages: 1) rapid eye movement (REM) sleep and 2) nonREM (NREM) sleep. REM sleep is a distinctive sleep stage that alternates with episodes of NREM sleep. The spontaneous NREM-REM sleep cycle in the rat takes about 12 to $20 \mathrm{~min}^{2-6)}$. Over the last four decades, most of the sleep research has focused on identifying relevant brain structures, neuronal networks, and their transmitters that are

\footnotetext{
\# To whom correspondence should be addressed.

(Tel) +82-43-261-2827; (Fax) +82-43-261-2827

(E-mail) kiwan@chungbuk.ac.kr
}

involved in the generation and regulation of NREM and REM sleep ${ }^{7-10)}$. Few studies have focused on understanding the possible mechanisms and modulating methods for the ultradian periodic occurrence of NREM-REM sleep.

Panax Ginseng, a well-known herbal medicine, has been used as a traditional medicine for thousands of years. Ginseng is now a popular and worldwide used natural medicine. Ginseng had a complex profile of activity. It is sometimes difficulty to reconcile on the available neurochemical reports on the plant. Ginseng has the effects of stabilizing and balancing the whole physiology ${ }^{11)}$. Ginseng has been used for treatment of insomnia clinically ${ }^{12)}$.

However, some controversial reports indicated that sleep disorders were most commonly experienced adverse events of ginseng ${ }^{13)}$. This study was undertaken to assess the role of ginseng in regulation sleep/wake fluctuation and architecture of sleep, and to determine possible mechanisms of ginseng on modulation of NREM/REM sleep. 
We also examined the amount of total sleep and wakefulness, and investigated power density changes in recorded electroencephalogram (EEG) of specific sleep-wake stage in freely moving rats.

\section{MATERIALS AND METHODS}

\section{Materials}

Six-year Korean red ginseng (KRG) was purchased from a market of Nong-Hyup in Cheongju area. KRG was extracted three times with $1,000 \mathrm{ml}$ of boiling water for 4 hours under reflux at the room temperature. This extract was subjected to filtration and concentration. Finally, ethanol extract of KRG was freeze-dried followed by evaporation of the remaining supernatants and used as the test samples.

\section{Animals}

Experiments were performed on 24 adult male Wistar rats (Samtako, Korea) weighing between 250 350 g. Rats were housed individually with food and water provided ad libitum under an artificial 12-h light/dark cycle (light on at 7:00) and at a constant temperature $\left(22 \pm 2^{\circ} \mathrm{C}\right)$. The rats were household in the departmental holding room for 1 week before testing. All the rats were maintained in accordance with the National Institute of Toxicological Research on the Korea Food and Drug Administration guideline for the care and use of laboratory animals.

\section{Surgery}

The animals were divided into 3 groups (control, 10 and $100 \mathrm{mg} / \mathrm{kg}$ of KRG) with 8 rats in each. Each rat was implanted with a transmitter (Data Sciences TA11CTAF40) for recording EEG and activity via telemetry as described previously ${ }^{14)}$. The body of the transmitter was implanted subcutaneously off midline and posterior to the scapula, and it was attached to the skin with 3 sutures for stabilization. Leads from the transmitter were led subcutaneously to the skull and the bare ends placed in contact with the dura through holes in the skull (A: 2.0 [Bregma], L: 1.5; P: 7.0 [Bregma], L: 1.5 contra-lateral). The electrodes were anchored to the skull with screws and dental cement. All surgical procedures were performed using stereotaxic apparatus. Surgical anesthesia was achieved with pentobarbital (50 mg/kg, ip).

\section{Data collection}

Following 7 day post-surgical recovery, telemetric recording of cortical EEG and activity were conducted using procedures similar to previous reports ${ }^{14)}$. For the
EEG signal, the gain of transmitters was set at $-0.5 /+0.5$ volts per/units $X 2$ and raw signals generated from the transmitter were in the range of $0.5-20.0 \mathrm{~Hz}$. The signals were processed by a Data Sciences analog converter and routed to an AD converter (Eagle PC30) housed in a PC class computer. The $\mathrm{AD}$ converter digitized the $\mathrm{EEG}$ and activity signals at $128 \mathrm{~Hz}$. The digitized data were transferred to the computer and displayed graphically by the program on the computer monitor. An on-line fast Fourier transformation (FFT) was performed on EEG data in every $2 \mathrm{sec}$ of data (256 samples) after a Hanning window treatment. The FFT analyses generated the power density values from 0.0 to $20.0 \mathrm{~Hz}$ at a resolution of 0.5 $\mathrm{Hz}$. The FFT data were further averaged in the range of 0 to $20 \mathrm{~Hz}$ for every $10 \mathrm{sec}$. The sleep data and FFT results were saved to the hard disk every $10 \mathrm{sec}$ for additional off-line analyses. Movement of the animal in relation to the telemetry receiver generated transistor-transistor logic (TTL) pulses that were collected and counted as a measure of activity. KRG was administered orally $10 \mathrm{~min}$ before EEG recording. Recording began at 9:00-12:00 AM (Fig. 1).

Wake

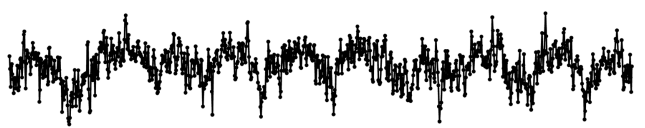

REM sleep
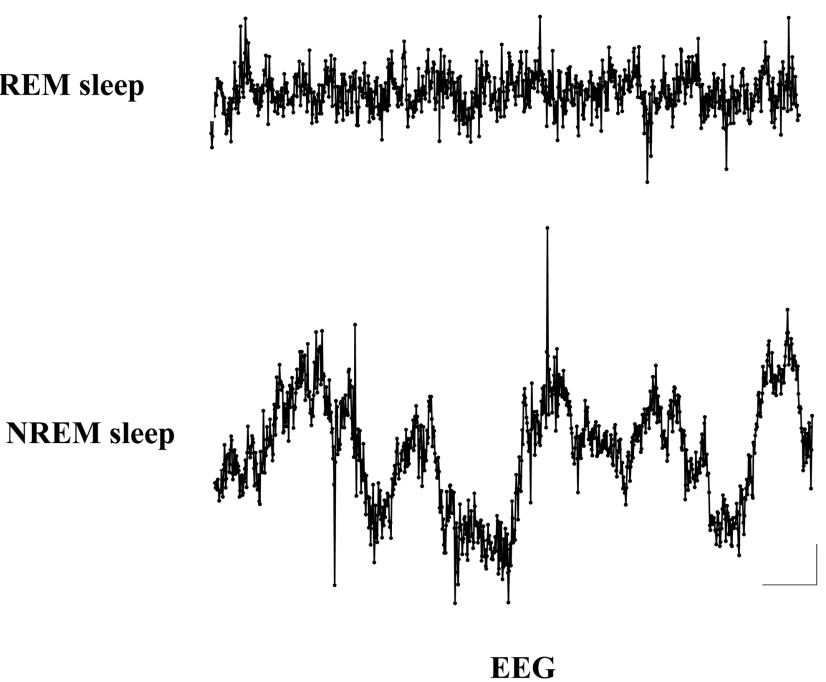

Fig. 1. Typical cortical EEG recordings of each stages of wakefulness, NREM and REM sleep in rats. EEG: electroencephalogram. Vertical bar: $50 \mu \mathrm{V}$, horizontal bar: $20 \mathrm{~s}$ in the panel of right corner. 
Determination of behavioral states and analysis in EEG power

The amount of time in wakefulness, NREM and REM sleep were determined from the digitized data on $10 \mathrm{sec}$ epochs using professional animal sleep analysis software SleepSign 2.1 (KISSEI Comtec Co Ltd. USA). Briefly, the software discriminates wakefulness as high-frequency lowamplitude EEG. NREM was scored based on the presence of spindles interspersed with slow waves in the EEG. EEG power during lower frequency $\delta$-wave $(0.75-4.5 \mathrm{~Hz}$ ) is significantly reduced in REM sleep and the range of $\theta$ wave activity $(5.0-9.0 \mathrm{~Hz}$, peak at $7.5 \mathrm{~Hz}$ ) was increased.

\section{Data analysis}

The time spent (min) in NREM, REM, and total sleep time (NREM+REM) were processed to obtain $12 \mathrm{~h}$ period totals for each rat. We further calculated the time of each recording spent in each sleep-wake state (wake, NREM, REM). The absolute EEG power during wakefulness, NREM, and REM were calculated in $0.5 \mathrm{~Hz}$ bins from 0.5 to $20 \mathrm{~Hz}$ for the entire $12 \mathrm{~h}$ records of each recording process. Afterwards, in each state, EEG power density in 3 selected frequency bands for wakefulness, NREM, and REM [ $\delta$-wave $(0.75-4.5 \mathrm{~Hz}), \theta$-wave $(5.0-9.0 \mathrm{~Hz})$ and $\alpha$ wave $(8.0-13.0 \mathrm{~Hz})]$ were evaluated.

\section{Statistical analysis}

All statistical analyses were conducted using SigmaStat software (SPSS, Inc.). One-way repeated measures of analysis of variance (ANOVA) procedures were used in the data analyses across days. After significant ANOVAs, post hoc comparisons of means were conducted with Tukey tests.

\section{RESULTS}

\section{Effects of KRG on sleep architecture}

No change in REM sleep was observed in all the tested rats during the $12 \mathrm{~h}$ recording time. While the amount of wake and NREM after KRG administration changed in the course of the experiment. KRG $10 \mathrm{mg} / \mathrm{kg}$ induced a significant decrease in total wake time and increase in NREM and total sleep. Similarly, KRG $100 \mathrm{mg} / \mathrm{kg}$ decreased total wake time and increased total sleep time, the NREM and REM sleep increased but not statistically significant (Fig. 2).

\section{Effects of KRG on EEG power density during NREM sleep}

During NREM, $\delta$-wave was reduced, $\alpha$-wave was increased, and $\theta$-wave power was not changed after 10 $\mathrm{mg} / \mathrm{kg}$ KRG administration. Differently, KRG $100 \mathrm{mg} / \mathrm{kg}$ did not induce any changes in EEG power density of all the 3 selected frequency bands (Fig. 3).

\section{Effects of KRG on EEG power density during REM sleep}

During REM sleep, KRG $10 \mathrm{mg} / \mathrm{kg}$ significantly decreased $\delta$-wave, increased $\alpha$-wave power density, without influencing power density of $\theta$-wave; KRG 100 $\mathrm{mg} / \mathrm{kg}$ did not induce any changes in EEG power density

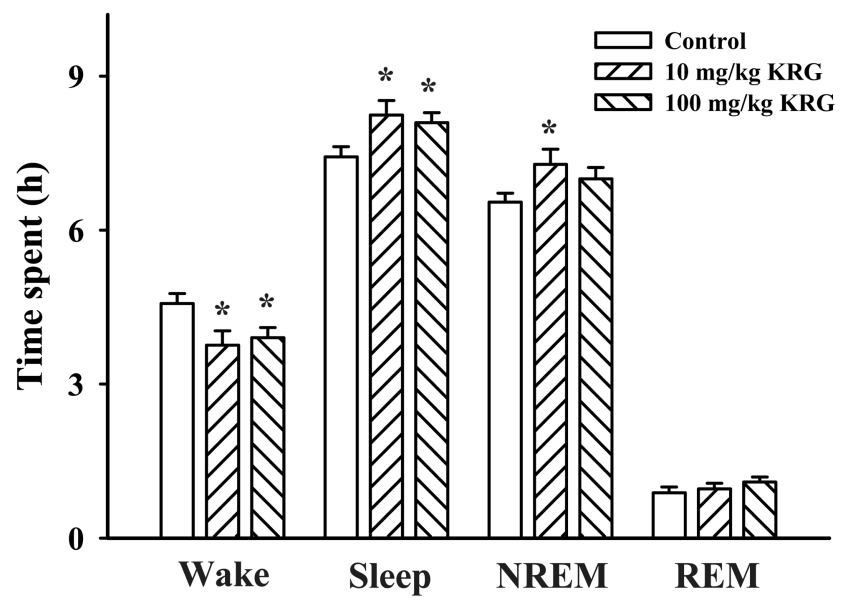

Fig. 2. Effects of KRG on sleep architecture. The data represents the mean $\pm \mathrm{SE}$ of time spent in sleep-wake state [Wake, Sleep (total sleep), NREM sleep, REM sleep]. * $\mathrm{P}<0.05$, significantly different from control.

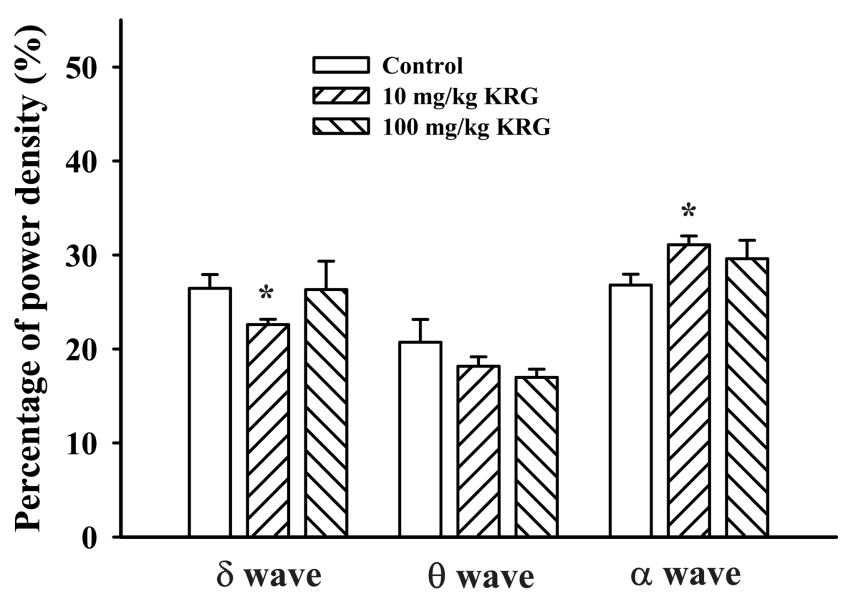

Fig. 3. Effects of KRG on EEG power density during NREM sleep. EEG power density in $\delta$-wave, $\theta$-wave and $\alpha$ wave for NREM were evaluated. The data represents the mean \pm SE of EEG power density in 3 selected frequency bands in NREM sleep stage. $* \mathrm{P}<0.05$, significantly different from control. 
of the 3 selected frequency bands (Fig. 4).

\section{Effects of KRG on EEG power density during wake time}

KRG (10 and $100 \mathrm{mg} / \mathrm{kg}$ ) did not change EEG power density of $\alpha$-wave. While low and high dose of KRG showed different effects on $\delta$ wave, KRG $10 \mathrm{mg} / \mathrm{kg}$ significantly decreased power density of $\delta$-wave, but KRG $100 \mathrm{mg} / \mathrm{kg}$ increased $\delta$-wave and decreased $\theta$-wave

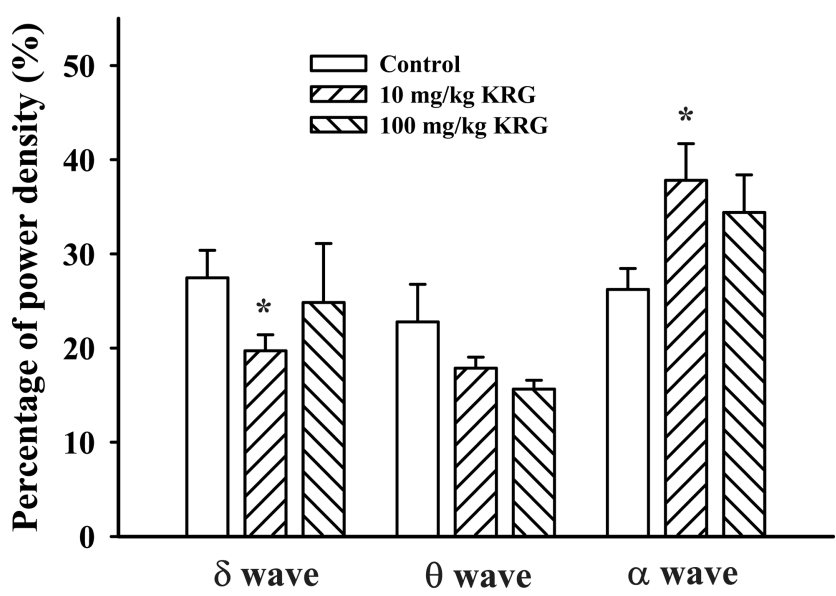

Fig. 4. Effects of KRG on EEG power density during REM sleep. EEG power density in $\delta$-wave, $\theta$-wave and $\alpha$-wave for REM were evaluated. The data represents the mean \pm SE of EEG power density in 3 selected frequency bands in NREM sleep stage. ${ }^{*} \mathrm{P}<0.05$, sig- nificantly different from control.

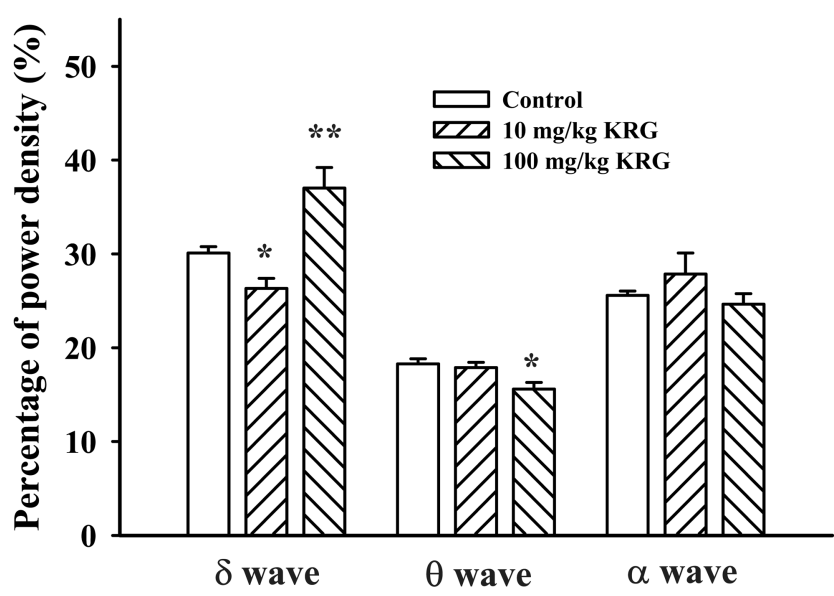

Fig. 5. Effects of KRG on EEG power density during wake time. EEG power density in $\delta$-wave, $\theta$-wave and $\alpha$-wave for wakefulness were evaluated. The data represents the mean \pm SE of EEG power density in 3 selected frequency bands in wake stage. ${ }^{*} \mathrm{P}<0.05$; $* * \mathrm{P}<0.01$, significantly different from control. power density during wake time (Fig. 5).

\section{DISCUSSION}

Our investigation indicated that KRG increased total and NREM sleep in rats, and decreased wakefulness. Panax ginseng is extensively used for a wide variety of clinical ailments and to improve general physical and mental wellbeing ${ }^{15)}$. People believe that ginseng has the sleep stabilizing and balancing effects. Our results are consistent with the belief that ginseng is effective in improving sleep and prove further that these effect are more specifically focused on NREM ${ }^{16)}$.

Over many years of research on the homeostatic regulation of sleep, it has been demonstrated that the homeostatic demand for its total amount and frequency depends on the specific activity patterns of the cortical EEG waves ${ }^{17-23)}$. A number of studies have suggested that the intensity of $\delta$-wave activity (or slow-wave activity; spectral power in the 0.75 to $4.0 \mathrm{~Hz}$ range) in the cortical EEG is the single most important process for the homeostatic regulation of NREM sleep ${ }^{17,19-25)}$. In support of this suggestion, studies have shown that the $\delta$-wave activity in NREM sleep typically declines in the course of the daily sleep period and increases in recovery sleep after a period of prolonged waking ${ }^{20,21,23,24)}$. Furthermore, it has also been reported that the $\delta$-wave activity is reduced in the subsequent NREM sleep after a nap and/or excess sleep 22,25). However, in our results, KRG increased NREM sleep with a decreased $\delta$-wave activity; therefore, it seems possible that KRG modulate sleep architecture with a similar way as physiological regulation in excess sleep.

Unlike NREM sleep, the EEG correlation for the REM sleep homeostatic process remained poorly understood. Some studies in humans suggested that the EEG activity of the $\alpha$-wave activity (frequency range of $8-13 \mathrm{~Hz}$ ) might be a marker of REM sleep homeostasis ${ }^{26}$. Yet, it is suggested that the $\alpha$-wave activity may not be involved in REM sleep homeostasis of rats ${ }^{27}$. Thus, it has been demonstrated clearly that the $\delta$-wave activity is still involved in the homeostatic regulation of REM sleep ${ }^{28)}$. In our study, KRG $10 \mathrm{mg} / \mathrm{kg}$ showed similar effects on NREM and REM effects, but significantly increased NREM and total sleep. REM. KRG $100 \mathrm{mg} / \mathrm{kg}$ did not change EEG power density of $\delta$-, $\theta$ - and $\alpha$-waves. Only, total sleep was increased. NREM and REM sleep were changed. These differences indicate that ginseng is more effective in lower dose in modulating of sleep, and it is more specifically related to the function of NREM sleep. 
Our results also found out that low dose $(10 \mathrm{mg} / \mathrm{kg})$ and high dose $(100 \mathrm{mg} / \mathrm{kg})$ of $\mathrm{KRG}$ showed different effects on $\delta$-wave and $\theta$-wave power density in wake time. It is another possible reason for the difference of sleep modulating effects between low and high doses of KRG.. In summary, the results of the current study demonstrated that KRG increases spontaneous sleep and NREM sleep and differently regulate the EEG spectra in REM and NREM sleep.

\section{ACKNOWLEDGEMENT}

This work was supported by the research grant of the Chungbuk National University in 2008.

\section{REFERENCES}

1. Datta, S. and MacLean, R. R. Neurobiological mechanisms for the regulation of mammalian sleep-wake behavior: reinterpretation of historical evidence and inclusion of contemporary cellular and molecular evidence. Neurosci. Biobehav. 31, 775-824 (2007).

2. Trachsel, L., Tobler, I., Achermann P. and Borbély, A.A. Sleep continuity and the REM-non REM cycle in the rat under baseline conditions and after sleep deprivation. Physiol. Behav. 49, 575-580 (1991).

3. Vivaldi, E. A., Ocampo, A., Wyneken, U., Roncagliolo, M. and Zapata, A. M. Short-term homeostasis of active sleep and the architecture of sleep in the rat. J. Neurophysiol. 72, 1745-1755 (1994).

4. Vivaldi, E. A., Wyneken, U., Roncagliolo, M., Ocampo, A. and Zapata, A. M. Measures of location and dispersion of sleep state distributions within the circular frame of a 12:12 light:dark schedule in the rat. Sleep. 17, 208-219 (1994).

5. Gottesmann, C. The transition from slow-wave sleep to paradoxical sleep evolving facts and concepts of the neurophysiological processes underlying the intermediate stage of sleep. Neurosci. Bibobehav. Rev. 20, 367-387 (1996).

6. Datta, S. and Hobson, J. A. The rat as an experimental model for sleep neurophysiology. Behav. Neurosci. 114, 1239-1244 (2000).

7. Alam, M. N., McGinty D. and Szymusiak, R. Neuronal discharge of preoptic/anterior hypothalamic thermosensitive neurons: relation to NREM sleep. Am. J. Physiol. 269, R1240-R1249 (1995).

8. Thakkar, M. M., Strecker R. E. and McCarley, R. W. Behavioral state control through differential serotonergic inhibition in the mesopontine cholinergic nuclei: a simultaneous unit recording and microdialysis study. J. Neurosci. 18, 54905497 (1998).

9. Pace-Schott, E. E. and Hobson, J. A. The neurobiology of sleep: genetics, cellular physiology and subcortical networks. Nat. Rev., Neurosci. 3, 591-605 (2002).

10. Blanco-Centurion, C., Xu, M., Murillo-Rodriguez, E., Gerashchenko, D., Shiromani, A. M., Salin-Pascual, R. J., Hof P. R. and Shiromani, P. J. Adenosine and sleep homeostasis in the basal forebrain. J. Neurosci. 26, 8092-8100 (2006).

11. Lee, S. P., Honda, K., Rhee, Y. H. and Inoué, S. Chronic intake of panax ginseng extract stabilizes sleep and wakefulness in food-deprived rats. Neurosci Lett. 111, 217-221 (1990).

12. Xiang, Y. Z., Shang, H. C., Gao, X. M. and Zhang, B. L. A comparison of the ancient use of ginseng in traditional Chinese medicine with modern pharmacological experiments and clinical trials. Phytother Res. 22, 851-858 (2008).

13. Coon, J. T. and Ernst, E. Panax ginseng: a systematic review of adverse effects and drug interactions. Drug Saf. 25, 323344 (2002).

14. Sanford, L. D., Yang, L., Liu, X. and Tang, X. Effects of tetrodotoxin (TTX) inactivation of the central nucleus of the amygdala (CNA) on dark period sleep and activity. Brain Res. 1084, 80-88 (2006).

15. Bahrke, M. S. and Morgan, W. R. Evaluation of the ergogenic properties of ginseng: an update. Sports Med. 29, 113133 (2000).

16. Rhee, Y. H., Lee, S. P., Honda, K. and Inoué, S. Panax ginseng extract modulates sleep in unrestrained rats. Psychopharmacology. 101, 486-488 (1990).

17. Borbély, A. A. A two process model of sleep regulation. Hum. Neurobiol. 1, 195-204 (1982).

18. Borbély, A. A., Baumann, F., Brandeis, D., Strauch, I. and Lehmann, D. Sleep deprivation: effect on sleep stages and EEG power density in man. Electroencephalogr. Clin. Neurophysiol. 51, 483-495 (1981).

19. Daan, S., Beersma, D. G. M. and Borbély, A. A. Timing of human sleep: recovery process gated by a circadian pacemaker. Am. J. Physiol. 246, R161-R178 (1984).

20. Dijk, D. J., Beersma D. G. M. and Daan, S. EEG power density during nap sleep: reflection of an hourglass measuring the duration of prior wakefulness. J. Biol. Rhyth. 2, 207-219 (1987).

21. Dijk, D. J., Brunner D. P. and Borbély, A. A. Time course of EEG power density during long sleep in humans. Am. J. Physiol. 258, R650-R661 (1990).

22. Werth, E., Achermann P. and Borbély, A. A. Brain topography of the human sleep EEG: antero-posterior shifts of spectral power. NeuroReport. 8, 123-127 (1996).

23. Franken, P., Chollet D. and Tafti, M. The homeostatic regulation of sleep need is under genetic control. J. Neurosci. 21, 2610-2621 (2001).

24. Tobler, I. and Borbély, A. A. Sleep EEG in the rat as a function of prior waking. Electroencephalogr. Clin. Neurophys- 
iol. 64, 74-76 (1986).

25. Feinberg, I., Maloney, T. and March, J. D. Precise conservation of NREM period 1 (NREMP1) delta across naps and nocturnal sleep: implications for REM latency and NREM/ REM alternation. Sleep. 15, 400-403 (1992).

26. Roth, C., Achermann, P. and Borbély, A. A. Alpha activity in the human REM sleep EEG: topography and effect of REM sleep deprivation. Clin. Neurophysiol. 110, 632-635 (1999).
27. Bjorvatn, B., Fagerland, S. and Ursin, R. EEG power densities $(0.5-20 \mathrm{~Hz})$ in different sleep-wake stages in rats. Physiol. Behav. 63, 413-417 (1998).

28. Shea, J. L., Mochizuki, T., Sagvaag, V., Aspevik, T., Bjorkum, A. A. and Datta, S. Rapid eye movement (REM) sleep homeostatic regulatory processes in the rat: changes in the sleep-wake stages and electroencephalographic power spectra. Brain Res. 1213, 48-56 (2008). 